\title{
Jacques Delaunay et les moines poètes Dampierre et Marconville dans les Epigrammata (1539)*
}

\author{
JOHN NASSICHUK
}

\begin{abstract}
In his little-known 1539 collection of epigrams, Jacques Delaunay (Jacobus Alnetus), a physician and canon of Saint-Étienne de Troyes, includes two important, and hitherto unnoticed, poetic exchanges between the monastic humanist poets Jean Dampierre and Nicolas de Marconville and himself. After a brief presentation of these two figures, the present article examines the principal themes and literary techniques characteristic of the poems that comprise the two poetic "correspondences" with Delaunay. Examination of these two separate sequences reveals several common, unifying themes. The relative (perceived) impoverishment of their poetic invention, and the deep anguish that it generates, constitutes a recurring motif, and a veritable preoccupation, for these poets, all of whom speak of the challenges posed by duties that distract them from cultivating the muse. Their collective meditation on these themes quietly elaborates a defense of the dignity of minor poetic genres such as the epigram in hendecasyllables or elegiac distichs, insofar as these genres often give voice to relations of friendship, love, and Christian solidarity.
\end{abstract}

Le recueil d'épigrammes latines signé Jacques Delaunay (Jacobus Alnetus, ?-1539?), médecin originaire de Vendôme, imprimé en 1539 aux presses troyennes de Jean Lecoq, ${ }^{1}$ comporte plusieurs pièces adressées aux hommes de lettres contemporains et notamment aux poètes. $^{2}$ La majeure partie des noms évoqués, comme ceux de Nicolas Bérauld, Guillaume et Jean Du Bellay, Étienne Dolet, Jean Salmon Macrin, Hubert Suzanne, Nicolas Bourbon, Clément Marot, François de

* L'auteur souhaite remercier l'équipe éditoriale de Humanistica Lovaniensia, les deux évaluateurs anonymes et le professeur Dirk Sacré, dont les nombreuses corrections lui ont permis d'améliorer la forme et le fond de ce travail. Il va de soi que toute erreur subsistante relève de la seule responsabilité de l'auteur.

1 Jacobi Alneti vindocinensis Epigrammata. Ad Joannem Bellaium Cardinalem amplissimum(Troyes: Jean Le Coq, 1539) [USTC 111142].

2 Voir John Nassichuk, "Les Epigrammata de Jacques Delaunay (1539) et les «poètes connus» à l'époque de François Ier", Bibliothèque d'Humanisme et Renaissance 82.1 (2020), 83-102. 
Sagon et Nicolas Denisot ont déjà fait l'objet d'études. ${ }^{3}$ Cette constellation de poètes connus témoigne en effet d'un certain engagement de l'auteur avec les milieux littéraires parisiens proches des humanistes. Bénéficiaires de l'influence de ces milieux cultivés, ils font preuve d'une envergure propre à accommoder une variété de connaissances en matière à la fois chrétienne et profane. Ils se montrent aussi, en général, adeptes d'une invention poétique informée par l'innutrition savante qui puise ses ressources dans les œuvres d'un grand nombre d'auteurs anciens et modernes. Plusieurs de ces poètes, en particulier Suzanne, Denisot et Macrin, ont laissé à la postérité des œuvres d'inspiration chrétienne sans que cette orientation thématique emblématise l'entier de leur production écrite. Leur maîtrise de l'éloquence savante formée sur le principe de la docta varietas constitue le noyau d'une production diverse et féconde, à l'image de celle des poètes humanistes du Quattrocento dont ils s'inspirent en mainte circonstance.

Une orientation plus rigoureuse dans le sens de la poésie chrétienne et dévotionnelle semble en revanche caractériser un autre groupe de poètes également reconnus du "cellérier et chanoine de Saint-Étienne de Troyes" que fut Jacques Delaunay, et dont la présence occupe une place tout aussi centrale au sein du recueil. ${ }^{4}$ Deux noms de moines, Jean Dampierre (14 ??-1550) et Nicolas de Marconville (14??- 154?), figurent non seulement parmi les lettrés destinataires des pièces de Delaunay, mais aussi à la tête de certaines pièces qui lui sont adressées. Dans ces cas, l'auteur et signataire de la collection présente à ses lecteurs une brève correspondance poétique entre ses confrères poètes et lui-même. Ce sont ces échanges avec Dampierre et Marconville, au cours desquels apparaissent des compositions des deux poètes moines peu étudiées jusqu'à présent, qui constituent l'objet de la présente étude. À la suite d'une brève présentation des auteurs concernés, il s'agira pour nous d'examiner les principaux thèmes et procédés littéraires qui caractérisent

${ }^{3}$ Pour des contributions récentes, voir notamment D. Speziari, La Plume et le pinceau. Nicolas Denisot, poète et artiste de la Renaissance (1515-1559) (Genève, 2016); P. Galand et al. (ed.), Nicolas Bérauld.Praelectio et commentaire à la Silve Rusticus d'Ange Politien (1515) (Genève, 2015); S. Laigneau-Fontaine (ed.), Nicolas Bourbon. Nugae (Bagatelles) 1533 (Genève, 2008).

4 Voir le poème de Nicolas Bourbon imprimé à la f. 46r du recueil sous le titre "Jacobi Alneti Vindocinensis Poetae et Medici doctissimi, Sancti Stephani Trecensis Cellarii et Canonici, huius opusculi Authoris Epicedium". Il s'agit en réalité d'une fausse rumeur de la mort de Delaunay, déplorée prématurément par Bourbon. Voir Nassichuk 2020 (as in n. 3), 96. 
les pièces tant de Delaunay que de ses interlocuteurs étroitement liés à l'univers monastique. Au cours de leur réflexion collective, des thèmes centraux émergent qui tendent à réunir l'ensemble du corpus. La pauvreté de l'invention poétique, à titre d'exemple, et l'angoisse qu'elle génère chez celui qui écrit, surgit à plusieurs reprises sous la plume de ces trois poètes. Delaunay et Marconville en particulier suggèrent que les exigences de la vie active, surtout dans les domaines de la médecine et du droit dont ils demeurent praticiens malgré leurs attaches aux milieux monastiques, tendent à compromettre la fécondité de la muse. Connexe à ce thème récurrent est celui, plus subtil, évoqué sur un ton ludique et enjoué, de la beauté insoupçonnée des objets humbles, y compris celle des poésies modestes. Dans la mesure où ils reviennent fréquemment sur ce thème de l'humilité de leurs vers, l'œuvre commune qu'élaborent les trois hommes constitue aussi une plaidoirie tranquille, sans déclarations ouvertement revendicatrices ni doctrinaires, en faveur de la dignité d'une écriture poétique pratiquée souvent de façon solitaire, par des poètes humanistes engagés dans la vie du siècle. ${ }^{5}$

\section{Les correspondants monastiques et contributeurs au recueil de Jacques Delaunay}

Nicolas de Marconville fut chantre de l'Abbaye Notre-Dame de Molesme et l'auteur d'un petit recueil de vers latins publié en $1537,{ }^{6}$ peu après le décès prématuré de François de Valois, duc d'Orléans. ${ }^{7}$ Le manuscrit 165 de la bibliothèque municipale d'Auxerre contient le compte rendu qu'il

5 Sur la vogue - et la louange - de ces genres "mineurs" à l'époque, voir l'article récent de P. Galand, "Les genres mineurs: une voie pour les parlementaires - poètes", in C. Gautier, E. Hénin, V. Leroux (ed.), Subversion des hiérarchies et séduction des genres mineurs (Leuven, 2016), 41-56.

6 Nicolai Marcomvilli Molismensis Coenobitae in ecclesiasticum illud, MEMENTO HOMO QUIA CINIS ES, et c. Elegiacum. | Eiusdem in Delphini obitum, et aliquot illustrium virorum, deploratio, cum diversis epigrammatis. (Paris: Louis Cyaneus, 1537).

7 Sur la déploration poétique de cet évènement survenu à la suite de ce qui fut vraisemblablement un accident, voir l'article de V.-L. Saulnier, "La mort du dauphin François et son tombeau poétique (1536)", Bibliothèque d'Humanisme et Renaissance 6 (1945), 50-97. Voir aussi, plus récemment, M. Clément, "Un geste poétique et éditorial en 1536: le Recueil de vers latins, et vulgaires de plusieurs Poëtes Françoys, composés sur le trespas de feu Monsieur le Daulphin", Réforme, Humanisme, Renaissance 62 (2006), 31-43; M.-A. Boitel-Souriac, "Le mythe du 'Dauphin-roi', l'exemple des funérailles littéraires de François de Valois (1536-1537)", Cahiers de la Méditerranée 77 (2008), 27-38. 
composa à l'intention du bien jeune Louis de Lorraine, abbé commendataire de Saint Germain et futur cardinal de Guise, de la visite des bénéfices dépendants de cette abbaye qu'il effectua le 26 juin 1542 ainsi que des "ordonnances de réformation" préparées quelques mois plus tard. ${ }^{8}$ Il est probable que l'importante fonction administrative qu'il remplit, selon le témoignage de ce document, dans l'intérêt de la réforme monastique correspond de près aux exigences lourdes de la vie active évoquées dans son échange poétique avec Jacques Delaunay. Après avoir loué, dans un des poèmes qu'il lui destine, la douceur de la vie monastique menée par son correspondant Marconville, Delaunay se réfère néanmoins plus d'une fois aux difficultés du métier de juriste qu'exerce celui-ci.

Quant à Jean Dampierre, ce recteur du couvent de la Madeleine-lèsOrléans fut connu et admiré des lettrés de l'époque et notamment des poètes humanistes d'expression latine. Juriste, lui aussi, ayant rempli des fonctions d'avocat au Parlement de Paris, il fut nommé aumônier du roi pendant les premiers moments du règne de François Ier et semble avoir siégé au Grand Conseil, d'après les souvenirs rapportés par Scévole de Sainte-Marthe, ${ }^{9}$ avant d'entrer en religion vers 1528 au plus tard. ${ }^{10}$ Connu pour sa maîtrise de l'hendécasyllabe latine, ${ }^{11}$ Dampierre est l'auteur d'un traité en quatre livres composé dans cette forme poétique, ${ }^{12}$ actuellement conservé dans le manuscrit 8349 de la Bibliothèque nationale de France, sur la condition des religieuses, leur disposition physionomique, médicale

8 Auxerre, Bibliothèque municipale , ms. 165: "Compte rendu par Nicolas de Marconville, chantre de Molesme, à Louis de Lorraine, abbé commendataire de SaintGermain, de la visite des prieurés et bénéfices dépendants de ladite abbaye, visite autorisée par lettres royales du 26 juin 1542; ordonnances de réformation rendues par Nicolas de Marconville (1542-1543, janvier)."

9 Scaevolae Sammarthani lucubrationum pars altera, qua continentur Gallorum doctrina illustrium, qui nostra patrumque memoria floruerunt Elogia (Paris: Pierre Durand, 1616), 29: “Ac huius quidem abunde comparatis inter celebriores magni Consilii patronos eminere iam coeperat..."

10 Voir sur cette séquence biographique les remarques de H. Meylan, "Bèze et les 'sodales' d'Orléans", in H. Meylan, D'Erasme à Théodore de Bèze (Genève, 1976), 139-144 et, plus récemment, la mise au point d'O. Pédeflous, "Macrin, Bourbon, Dampierre devant 1'Affaire des Placards (1534)", Papers in French Seventeenth-Century Literature 71 (2009), 459-474.

11 Scèvole de Sainte-Marthe, Elogia, 29: "Nam in hendecasyllabis, quorum aliquot per manus politiorum circumferuntur, Macrino ipso mollior ad Catullum proxime accedit."

12 Meylan 1976 (voir la n. 10), 143, suivant J. Boussard, suggère à tort que le traité de Dampierre aurait été composé en "un poème de quatre mille vers hexamètres". En réalité, le grand opus de Jean Dampierre fut rédigé en hendécasyllabes phaléciens. 
et spirituelle, et la bonne manière de les guider dans l'existence qu'elles mènent au couvent. ${ }^{13}$ Un tel thème, élaboré de façon détaillée, montre bien que l'entrée en religion n'a guère entravé l'invention de l'auteur, même si celui-ci fut plutôt exceptionnel parmi ses confrères pour sa participation aux salons littéraires d'Orléans. ${ }^{14}$

Alors qu'un moine réformé comme Jean Dampierre n'affiche certainement aucune hostilité à l'égard des belles-lettres, la tendance générale dans les milieux monastiques pendant ces années suggère néanmoins une certaine méfiance envers l'humanisme lettré. ${ }^{15}$ Les savants qui figurent dans leurs rangs, tels Dampierre ou encore le célestin Denys Lefebvre, autre ami de Delaunay, ${ }^{16}$ sont souvent des humanistes qui, ayant déjà acquis une formation littéraire à l'extérieur du monastère, ne renoncent que tardivement à la vie du siècle. ${ }^{17}$ Leur expérience du monde comprend naturellement des relations dans les cercles richement nourris de la poésie de l'Antiquité et de l'humanisme. L'intérêt grandissant des milieux laïcs pour la littérature spirituelle de langue française, et pour les pratiques dévotionnelles personnalisées, tend aussi à renforcer la proximité entre modes et genres d'écriture exercés à l'intérieur comme à l'extérieur des monastères. ${ }^{18}$ Cette complicité trouve un écho contemporain parmi les auteurs de langue latine dans la thématique érasmienne de l'amitié qui favorise la convergence des modalités rhétoriques héritées de l'Antiquité tant chrétienne que

13 Paris, Bibliothèque nationale de France, ms. Latin 8349: Joannis. [sic] Dampetri phaleuciorum libri quatuor de institutione eius, cui sunt Virgines instituendae; quorum duo priores ad corporis salutem, posteriores duo ad curam animarum.

14 J.-M. Le Gall, Les moines au temps des réformes (Paris, 2001), 211.

15 Le Gall 2001 (voir la n. 14), 214: "Les réformateurs monastiques ont le sentiment d'édifier un rempart contre les éventuelles dérives dangereuses et paganisantes de l'humanisme naissant. Bien que peu développé en France du Nord, l'humanisme profane est pourtant craint."

16 Une épigramme de Jacques Delaunay, en hendécasyllabes, apparaît en préface au poème didactique de Denys Lefèbvre sur l'Immaculée Conception de Marie, publié chez le même éditeur troyen. Voir: Fratris Dionysii Fabri Vindocinensis Celestini, de purissimo Mariae Virginis conceptu: versibus intercalariis Liber unus distinctus. (Troyes: Jean Le Coq, [1520]) [USTC 111133].

17 A. Renaudet, Préréforme et humanisme à Paris pendant les premières guerres d'Italie (1494-1517) (Paris, 1953), 517; V.-L. Bourrilly, Guillaume Du Bellay, seigneur de Langey (1491-1543) (Paris, 1905), 9.

18 A. Passot-Mannooretonil, Poètes et pédagogues de la Réforme catholique (Paris, 2019). 
païenne. ${ }^{19}$ En vérité, la thématique de l'amitié monastique réunit désormais les confrères lettrés au sein de cet univers culturel, comme le montre l'exemple remarquable du savant bénédictin Trithemius, d'abord abbé de Sponheim, ensuite évêque de Würzburg..$^{20}$ Le modeste échange poétique préservé dans le recueil de 1539, qui réunit Jacques Delaunay aux moines poètes Nicolas de Marconville et Jean Dampierre, constitue un nouvel exemple phare de cet esprit de corps lettré qui règne largement parmi les humanistes, y compris dans les milieux éloignés des écoles parisiennes et de la Cour.

L'esprit de corps en vigueur dans les milieux des humanistes latinisants se manifeste, en maint cas, par la voie d'une correspondance latine à travers laquelle les champions érudits des "bonnes lettres" élaborent un dialogue qui donne voix à des valeurs partagées. ${ }^{21} \mathrm{Si}$ la correspondance des grands humanistes prosateurs, tels Erasme, Salutati, Bruni et Budé constituent sans doute le principal modèle de cette pratique culturelle qui puise ses modèles chez des auteurs anciens comme Cicéron, Sénèque, Augustin et Jérôme, les auteurs d'épigrammes antiques, notamment Catulle et Martial, ont légué à leurs héritiers modernes un exemple de l'échange dialogué en vers, à caractère souvent spirituel et ironique, qui fut destiné à connaître un essor impressionnant parmi les auteurs de vers latins contemporains des prosateurs les plus éminents. Les auteurs d'épigrammes latines que Lucien Febvre a fameusement surnommés les "Apollons de Collège" exercent, pour la plupart, le métier de professeur dans les collèges parisiens. ${ }^{22}$ Ils ont laissé un corpus significatif de pièces adressées aux collègues et aux amis, parmi lequel il est possible de restituer des échanges, une sorte de correspondance en vers, qui témoignent justement de la conscience qu'ils avaient de leur mission commune. Certes plus humble que celle des grands humanistes de Cour, cette mission collective les réunit dans l'amour des lettres

19 K. Eden, Friends Hold All Things in Common. Tradition, Intellectual Property, and the Adages of Erasmus (New Haven - London, 2001), 142.

20 N.L. Brann, The Abbot Trithemius (1462-1516). The Renaissance of Monastic Humanism (Leiden, 1981), 229-230.

21 Voir notamment les études réunies dans F. Wild (ed.), L'art de la lettre humaniste (Paris, 2004).

${ }^{22}$ L. Febvre, Le problème de l'incroyance au XVIe siècle. La religion de Rabelais (Paris, 1947), 21. 
latines. ${ }^{23}$ Sans constituer une véritable correspondance, au sens formel du terme, l'échange qui se dégage de ce corpus de pièces parfois ironiques et allusives montre en effet, à plusieurs endroits, l'existence d'un procédé épistolier, d'un "pacte de la correspondance" 24 implicitement reconnu de part et d'autre. Or, les échanges poétiques entre Jacques Delaunay et ses correspondants Marconville et Dampierre, préservés dans le recueil publié sous le nom du premier, présentent à la fois des épigrammes brèves et allusives et certaines pièces plus longues, qui ressemblent à de véritables épîtres, dans lesquelles les auteurs se permettent des réflexions plus détaillées que ne sont celles des "Apollons". Leur vivacité ironique et l'esprit de répartie badin qui les caractérise donnent sans doute un écho lointain du genre de l'échange poétique et fictif, abondamment représenté dans la littérature du Moyen Âge latin et vernaculaire, le Streitgedicht, ${ }^{25}$ sans toutefois reproduire les caractéristiques formelles de ce type de dialogue élaboré avec humour sur le mode de la disputation. ${ }^{26}$ Ces échanges entre Delaunay et ses correspondants, qui se construisent dans le contexte de l'univers monastique et non celui des écoles parisiennes, présentent des réflexions où la tonalité badine donne parfois lieu à des épanchements sur les thèmes de l'amitié, de la poésie et des contraintes de la vie active au sein même des lieux consacrés à la contemplation spirituelle.

23 Voir à ce sujet le récent volume collectif publié par M. Ferrand et N. Istasse (ed.), Nouveaux regards sur les 'Apollons de Collège'. Figures du professeur humaniste dans la $1^{\text {ère }}$ moitié du XVIe siècle (Genève, 2014).

24 L'expression est de J.-E. Girot, Marc-Antoine Muret. Des Illes Fortunées au rivage romain (Genève, 2012), 270-271.

25 Voir sur ce genre largement répandu en Europe à partir de l'Antiquité tardive, la monographie fondatrice de H. Walther, Das Streitgedicht in der lateinischen Literatur des Mittelalters (München, 1920), qui montre les influences multiples des genres poétiques anciens, de la Fable à l'églogue virgilienne, dans la constitution de la scène dialoguée du Streitgedicht. Pour une discussion plus récente de la même problématique de l'histoire littéraire, voir J.O. Fichte, P. Stotz et al., Das Streitgedicht im Mittelalter (Stuttgart, 2019).

26 Signalons toutefois que plusieurs pièces individuelles dans le recueil de Delaunay affichent des traits de ce genre d'échange poétique basé sur la répartie badine et spirituelle, où les interlocuteurs renchérissent avec énergie et ingénuité les uns sur les autres. Voir ainsi "De Oenophilo et Abstemio litigantibus iudicium" (f. 7v) et "Interlocutores. Scientia. Stultus. Ambitiosus. Avarus. Studiosus” (f. 24v-25r). 


\section{Nicolas de Marconville}

Le recueil de Jacques Delaunay contient quatre pièces adressées à Nicolas de Marconville, poète d'expression latine et chantre de l'Abbaye NotreDame de Molesme. Il s'agit d'un corpus limité, qui constitue la totalité des renseignements préservés sur les rapports entre les deux hommes. La première de ces pièces apparaît entre deux poèmes identiques de longueur et de forme -22 vers hendécasyllabiques - destinés aux humanistes Nicolas Bérauld et Jean Salmon Macrin. Il s'agit d'une épigramme de dix-huit vers, composée en distiques élégiaques, dans laquelle Delaunay chante l'éloge de son destinataire qu'il identifie comme un moine poète actuellement - et bienheureusement - en résidence à Molesme. Dès l'ouverture du poème, l'auteur affirme solennellement que les Muses habitent désormais le célèbre monastère, s'étant réfugiées auprès de leur protégé Marconville, et qu'elles trouvent leur bonheur à ses côtés dans des lieux si propices à l'inspiration:

Errabam nuper circum delubra Molismi, pascis ubi pullum dive Roberte gregem.

Haec errare novem vidi per templa sorores, Quas bonus Ascreis vidit alumnus agris.

Certabant cantu inter se, floresque legentes Texebant facili florea serta manu.

Has adeo Musas, dixi quibus obvius, esset Ut mihi tam dulcis cognita causa soni.

Quis canor? et quis vos dux aedibus excipit istis?

Florea cui vultis serta parare Deae? ${ }^{27}$

L'apostrophe de Robert de Molesme, fondateur de l'abbaye de Molesme et de l'ordre Cistercien, donne dès le premier distique de l'épigramme un indice clair du contexte dans lequel Nicolas de Marconville poursuit les voies de l'invention poétique. Déployant le motif conventionnel du déplacement des Muses descendues de l'Hélicon, l'auteur présente son

27 Jacobi Alneti vindocinensis Epigrammata (as in n. 1), f. 6r-v: "Je me trouvais naguère devant l'église de Molesme, / Où tu fais paitre, saint Robert, ton petit troupeau. / A travers ce temple j'ai vu se promener neuf sœurs / Que ton bon élève a vues dans les champs d'Ascrée. / Elles rivalisaient de chant entre elles, et en cueillant des fleurs / Elles tissaient d'une main légère leurs guirlandes fleuries. / Venant au-devant de ces muses, je leur dis de me / Faire connaître la cause d'un son aussi doux. / Quel est ce chant? Quel est le chef qui vous accueille dans cet édifice? / Pour qui préparez-vous, déesses, ces guirlandes fleuries?" 
ami Marconville comme un poète remarquable, et l'abbaye de Molesme comme un véritable lieu sacré de l'inspiration poétique. Il raconte que lors d'une visite récente à l'abbaye, l'une des muses leva la voix pour confirmer devant lui l'identité de leur guide et coryphée - Marconville qui les séduisit naguère par la haute qualité de ses chants. ${ }^{28} \mathrm{La}$ première image du moine est donc celle d'un poète exceptionnel, entouré de ses muses dans un lieu idyllique où il se consacre principalement aux œuvres de la foi et à la composition des vers. ${ }^{29}$ Delaunay décrit cette scène en usant d'une langue qui évoque déjà le contexte festif et pastoral, comme en témoigne l'emploi de l'expression florea serta dans le deuxième hémistiche du pentamètre. ${ }^{30}$ La double occupation spirituelle et poétique devient aussi le lieu d'une convergence harmonieuse, où la méditation sacrée nourrit la confection de vers latins à la fois bien tournés et édifiants.

Cette description de Marconville comme poète entièrement dévoué à son art ne constitue que le premier élément du portrait complexe qui émergera de l'ensemble des pièces que Jacques Delaunay lui adresse. La deuxième est une épigramme de 14 vers composée en distiques élégiaques, où l'auteur ajoute encore des précisions concernant le type de poésie que compose son ami. Marconville, affirme-t-il, écrit des vers à caractère moral, dans lesquels il décrit les mœurs du siècle :

Marcomville bonum quis te neget esse poetam?

Cum tibi Castalio carmen ab amne fluat,

28 Jacobi Alneti vindocinensis Epigrammata (as in n. 1), f. 6v: "Una puellarum, cui laurea serta paramus / Est Marconvillus ductor et hospes ait. / Has noster sacras qui duxit Alumnus in aedes / Posthabito modulis nos Helicone suis. / Cuius honorata cinguntur tempora lauro / Quem sacer insignit relligionis honor. / Cantamus pariter cecinit quae carmina, cantu / Ex illo nostrum tendit ad astra melos." ("Une des jeunes filles répondit: celui pour qui nous tressons ces / Guirlandes de laurier est Marconville, notre guide et notre hôte. / C'est notre enfant qui nous conduisit dans cet édifice sacré / Avec ses mélodies, puisque nous l'avons préféré à l'Hélicon. / Ses tempes sont couronnées du laurier d'honneur, / C'est l'honneur sacré de la religion qui le distingue. / Chantons de concert les airs qu'il chanta, à partir de son chant / Notre mélodie monte jusqu'aux astres.")

29 Delaunay s'approche en cela du lieu commun, rencontré à l'époque chez des auteurs laïcs, qui idéalise le milieu monastique comme lieu d'inspiration. Voir PassotMannooretonil 2019 (voir la n. 18), 502-503.

30 Cf. Mart. Epigr. 8.77.4 et la construction presque identique chez Callimachus Experiens, Carmina, 7.47-48: "Quam iuvat et festis dominam spectare diebus, / Cum texit nivea florea serta manu!" 
Quo varios hominum mores describere tentas, Ut notat in nostra picta tabella domo. ${ }^{31}$

Delaunay évoque ici un "tableau peint" visible dans sa maison ("in nostra... domo"), dont l'image touche au thème de la diversité des mœurs des hommes. Se réfère-t-il à son propre domicile, ou plus généralement à l'abbaye Saint-Étienne de Troyes dont il est chanoine? Le motif privilégié, on le voit, dans ces vers n'est autre que celui du "théâtre du monde", l'auteur livrant un tableau des iniquités de la vie contemporaine ou, plus précisément, de l'existence terrestre empoisonnée par le mal et par la souffrance. Pour ce faire, il emploie une expression, varios hominum mores, qui, inattestée chez les poètes anciens, apparaît au moins deux fois chez un humaniste contemporain, notamment dans l'Art poétique de Marco Girolamo Vida, lorsque celui-ci décrit les diverses grandeurs de Virgile $^{32}$ et l'utilité de la poésie savante nourrie d'une connaissance profonde des mystères de la nature tant des hommes que des animaux. $^{33}$ Delaunay évoque dans cette description, selon toute vraisemblance, le contenu particulier du recueil que Marconville fit paraître en 1537 à la suite du décès du dauphin François de Valois. Marconville déploie dans ce recueil une rhétorique de la consolation, fortement axée sur le thème de la "misère de l'homme" souvent attesté dans l'éloquence latine morale à la suite du grand traité De miseria condicionis humanae du pape Innocent III. ${ }^{34}$ Maintes fois revisité par les humanistes du Quattrocento, le thème connut une vogue en France pendant les premières décennies du XVIe siècle, comme en témoigne le succès éditorial de la longue élégie de Simon Nanquier, auteur monastique contemporain de Robert Gaguin, intitulée De lubrico temporis curriculo deque hominis miseria [...], qui parut en plusieurs

31 Jacobi Alneti vindocinensis Epigrammata (as in n. 1), f. 13r: "Qui nierait, Marcomville, que tu sois un bon poète? / Puisque chez toi le chant coule de source castalienne. / Par ce moyen tu cherches à décrire les vicissitudes humaines, / Ce que montre le tableau peint dans notre demeure."

32 M.G. Vida, Ars poetica, 1. 389: "Profuit et varios mores hominumque, locorumque / Explorasse situs...".

33 M.G. Vida, Ars poetica, 2.459: "Hinc varios moresque hominum moresque animantum".

34 Voir sur cette vaste thématique la monographie toujours utile de C. Trinkaus, Adversity's Noblemen. The Italian Humanists on Happiness (New York, 1940). Plus récemment, J. Bartuschat, "Il 'De miseria humane conditionis' e la letteratura didattica delle lingue romanze", in Andrea Sommerlechner (ed.), Innocenzo III. Urbs et orbis (Rome, 2003), 340-351. 
éditions successives à partir de sa publication en 1498 lors du décès de Charles VIII. ${ }^{35}$

Après ces deux premières pièces, la prochaine épigramme adressée à l'habitant de Molesme apparaît aux feuilles 20r-21v et constitue le début d'une petite série de cinq poèmes qu'échangent les deux auteurs, dont le premier et le dernier sont de Delaunay, ${ }^{36}$ les trois intermédiaires de Marconville. ${ }^{37}$ Dans le premier de ces poèmes Delaunay répond avec énergie, en l'espace de 96 hendécasyllabes, à la demande de son ami qui lui réclame un poème, sollicitation dont le texte, si jamais il fut rédigé, n'apparaît pas au sein de ce recueil. Pour toute réponse, Delaunay prononce un adieu amer à la poésie; accablé par son travail de médecin, il dit ne plus trouver le temps d'écrire. C'est ici qu'apparaît, pour la première fois, la note pessimiste qui reviendra à plusieurs reprises dans ses vers, concernant la possibilité de mener de front une carrière dans la vie active et une activité de poète. La poésie, explique-t-il, malgré tout son dévouement ne lui a jamais vraiment servi:

Omnem qui lapidem novem colendis

Musis (quas tenero secutus ungue)

Movi, nil retuli tamen lucelli.

De me nil meritae fuere Musae;

Musae sunt steriles, inutilesque. ${ }^{38}$

Ces vers ouvrent une plainte conventionnelle, sur la difficulté de vivre de sa plume à une époque où les mécènes généreux se font rares. ${ }^{39} \mathrm{Ce}$ thème déjà ancien du malheur des hommes de lettres a connu un renouveau

35 De lubrico temporis curriculo de hominis miseria. Necnon de funere cristianissimi Regis Caroli octavi (Paris: Antoine Dénidel pour Robert Gourmont, 1498).

36 "Meo Nicol. Marcomvillo Molismeni Coenobitae", f. 20r-21v; "Meo Marcomvillo", f. $22 \mathrm{v}-23 \mathrm{r}$.

37 "Nic. Marcomvillus, suo Jac. Alneto", f. 21v-22r; "Ad eundem", f. 22r; "Ad Alnetum", f. 22r-v.

38 Jacobi Alneti vindocinensis Epigrammata (as in n. 1), f. 20 r: "Moi qui déplaçai toute pierre pour cultiver / Les neuf Muses (que je suivis depuis ma prime / Jeunesse), je n'en ai pas pour autant tiré le moindre profit. / À moi, les Muses ne m'ont accordé aucun bienfait; / Les Muses sont stériles et sans intérêt."

39 Jacobi Alneti vindocinensis Epigrammata (voir la n. 1), f. 20r: "Tempestate lucri suis in ista / Nil cultoribus afferunt Camaenae. / Multo Gallia vate gloriatur: / Rarus Tuscus Eques, Poeta multus." (“A cette époque de lucre les Camènes / N'apportent rien à ceux qui les cultivent. / La France se vante de maint prophète: / Rare est le chevalier étrusque, nombreux les poètes.”) Cf. notamment la cinquième églogue des Adulescentia de Baptista Mantuanus. 
éclatant sous la plume des humanistes pendant la première moitié du seizième siècle, comme en témoigne le dialogue latin De literatorum infelicitate, que Piero Valeriano Bolzano a composé au cours des années qui ont suivi le sac de Rome par les forces impériales en mai, $1527 .{ }^{40} \mathrm{En}$ France, la célèbre élégie de l'humaniste écossais George Buchanan sur les misères de l'enseignement de lettres à Paris, Quam misera sit conditio docentium literas humaniores Lutetiae, publiée pour la première fois en 1567 mais composée vraisemblablement bien auparavant, a formulé avec élégance la thématique familière aux humanistes formés dans les écoles parisiennes. Joachim Du Bellay devait la revisiter dans sa paraphrase intitulée "L'Adieu aux Muses pris du latin de Buccanan", imprimée dès 1552 au sein des Euvres de l'invention de l'auteur. ${ }^{41}$ Puisant ainsi dans une tradition qui remonte à Horace ${ }^{42}$ et Martial, ${ }^{43}$ le poète vendômois accommode la réflexion sur les difficultés de la vie littéraire au contexte particulier d'un échange avec le moine Marconville. Delaunay déclare avoir déjà fourni de gros efforts dans le domaine des lettres; il souhaite se consacrer désormais à des labeurs plus utiles, qui lui permettront de préparer sa retraite. "Telle est bien la règle, remarque-t-il avec ironie, que je dois maintenir." 44 Bien différente serait selon lui la situation de Marconville qui, dans l'abbaye de Molesme, jouirait d'une sérénité apte à bien nourrir l'invention poétique:

Marcomville opis est tuae novenas

Exercere Deas, linenda cedro

Clausi scribimus. Intimos recessus

Et conclavia postulant Poetae.

Sunt grata ocia floridis in hortis,

40 K. Gouwens, "Life-Writing and the Theme of Cultural Decline in Valeriano's De Litteratorum Infelicitate", The Sixteenth Century Journal27.1 (1996), 87-96.

41 Voir sur cette paraphrase l'article de N. Catellani-Dufrêne, "'L'Adieu aux Muses': Joachim Du Bellay traducteur de Buchanan", Bibliothèque d'Humanisme et Renaissance 69.2 (2007), 425-433.

42 Hor. Ep. 2.1.1-17.

43 Mart. Epigr. 8.55.9.

${ }^{44}$ Jacobi Alneti vindocinensis Epigrammata (voir la n. 1), f. 20v: "Haec victus ratio est habenda nobis." 
Ast dat gratius ocium Molismus

Secura tibi cogitatione. ${ }^{45}$

La maison religieuse fondée par Robert est de nouveau désignée dans ces vers comme un lieu de recueillement spirituel qui n'exclut pas le labeur de l'invention lettrée. Un écho de la Lettre aux Pisons - linenda cedro...scribimus - reflète ici l'esprit de méditation consciemment littéraire qui anime cette composition. ${ }^{46}$ En décrivant ainsi de façon idéalisée l'otium savant et inspiré dont jouirait le résident de Molesme, Delaunay se rapproche également d'un passage des Tristes, dans lequel Ovide évoque la tranquillité d'esprit nécessaire au poète. ${ }^{47}$ Dans ces conditions idéales, le moine studieux peut bénéficier pleinement des richesses de la vie contemplative:

Hic te Relligio facit quietum, Quae mundi tumidis caret procellis, Quae vitam facit esse suaviorem, Quae curas abigit molestiores. Hic dum quid meditaris, inquietat Nemo, aut te abstrahit a remissione. ${ }^{48}$

Si les soucis inhérents à la vie active n'interrompent jamais la contemplation studieuse du poète monastique, Delaunay rappelle à son ami que la vieillesse vient surprendre rapidement ceux qui, comme luimême, exercent une fonction dans le monde. Aussi aspire-t-il désormais à imiter la fourmi qui prépare ses jours d'hiver en travaillant diligemment

45 Jacobi Alneti vindocinensis Epigrammata (voir la n. 1), f. 20v- 21r: "Marconville, il t'appartient bien de t'occuper / Des neuf déesses et, enfermé chez moi, j'écris des choses / Dignes d'être enduits de l'huile de cèdre, car aux Poètes / Il faut des retraits intimes et des chambres fermées. / Agréables sont mes loisirs dans des jardins en fleur, / Mais Molesme te donne un repos encore / Meilleur, grâce à la méditation libre de tout souci."

46 Hor. Ars 330-332: “An, haec animos aerugo et cura peculi / Cum semel imbuerit, speramus carmina fingi / Posse linenda cedro et levi servando cupresso?"

47 Cf. Ov. Trist. 1.1.41-44: "Carmina secessum scribentis et otia quaerunt: / Me mare, me venti, me fera iactat hiems. / Carminibus metus omnis abest: ego perditus ensem / Haesurum iugulo iam puto iamque meo."

48 Jacobi Alneti vindocinensis Epigrammata (voir la n. 1), f. 21r: "Là, elle te procure de la paix, la vie religieuse, / Car elle est dépourvue des bourrasques gonflées du monde, / Elle fait que la vie a plus de douceur / Et elle chasse au loin les soucis bien pénibles. / Là, pendant que tu médites sur quelque sujet, personne / Ne te dérange, ni ne t'arrache à ta retraite." 
durant l'été. ${ }^{49}$ L'explication morale aux allures ésopiques, fondée sur l'exemple de la fourmi laborieuse, ne devait pas sembler étrange à un destinataire qui menait la vie du repos religieux, d'autant que la leçon avancée par Delaunay, qui loue dans la fourmi non l'industrie harmonieuse et l'esprit communautaire, mais bien les vertus de l'initiative individuelle et de la prudence, apparaît aussi dans des textes contemporains sur la vie monastique. Barthélemy de Loches, à titre d'exemple, dans un traité sur "la vie active et contemplative" publié en 1523 , cite avec approbation l'exemple de la fourmi prudente. ${ }^{50}$

La première réponse de Marconville est une pièce de 23 hendécasyllabes, la plus longue des trois épigrammes consécutives adressées à Delaunay, dans laquelle il se montre enchanté de la réponse de son correspondant. Il implore néanmoins celui-ci de ne pas renoncer à la poésie, malgré les difficultés de sa situation. Delaunay, affirme-t-il, ne saurait se priver du plaisir que les muses lui procurent, car ces muses lui sourient tout de même en le couronnant de laurier sinon de richesses. Il doit absolument se compter parmi les soldats qui suivent leurs étendards, car vers quel autre camp va-t-il se diriger? ${ }^{51}$ L'épigramme suivante présente, l'espace de deux distiques élégiaques, l'éloge du poète et médecin Delaunay, doublement fidèle à la vocation apollinienne. ${ }^{52}$ Finalement, dans la dernière épigramme qui lui est attribuée, Marconville emprunte le discours de la modestie excessive, voire de l'ironie pessimiste à l'égard de ses propres talents. Ses observations sur l'importance de la tranquillité pour ceux qui composent des vers, semblent confirmer tout à coup la résignation à laquelle Delaunay donnait voix dans son adieu à la poésie. Elles soulignent à nouveau l'impossibilité de tenir ensemble deux exigences difficilement conciliables, de la vie active, d'une part, et, d'autre part, de la liberté contemplative si nécessaire au travail des lettres. Le milieu des juristes, dans lequel

49 Jacobi Alneti vindocinensis Epigrammata (voir la n. 1), f. 21v: "Ut formica suo pigros labore / Exhortans medio legit sub aestu / Fruges, queis Hyemis pericla vitet, / Sic rebus vegeto labore partis / Ut tempus foveatur hoc senile / Attendo studio, anxiave cura." ("Tout comme la fourmi, exhortant les paresseux / Par son labeur, cueille au milieu de l'été les fruits / Qui permettent d'éviter les périls de l'hiver, / Ainsi les produits de mon labeur énergique, / Qui doivent soulager ce temps de ma vieillesse, / Je les attends avec impatience, dans un souci plein d'angoisse.")

50 F. Nicolai Barptholemaei, Lochiensis Doctoris De Vita activa et contemplativa. Liber unus (Paris: Pierre Vidoue, 1523) [USTC 184288 ], f. b.i.r-v.

51 Jacobi Alneti vindocinensis Epigrammata (voir la n. 1), f. 22r: "Mutatis clypeis, tubisque versis?"

52 Jacobi Alneti vindocinensis Epigrammata (voir la n. 1), f. 22r. 
Marconville, inspecteur des maisons religieuses reformées, exerce ses fonctions, comporte inévitablement des obligations lourdes qui minent la tranquillité de l'esprit. Il se peut bien que certains sachent composer de "doux poèmes" au moment même où ils se trouvent embrouillés dans la tension d'un procès légal, mais il est difficile de retrouver les douceurs de l'otium lorsqu'on est constamment sous la pression de la vie active. Le grand Homère lui-même, conclut Marconville, n'aurait su écrire ses épopées s'il n'avait pas été libre des mille tracasseries du negotium:

Ni curis vacuum sit intimum cor,

Et mens sit nebulis serena pulsis,

Vix illos numeros Homerus ille

(Qui fons ingenii, parensque fertur)

Totos Nestoris eruet per annos. ${ }^{53}$

A la suite de cette déclaration mélancolique, c'est maintenant Marconville qui implore Delaunay de ne plus faire l'éloge de ses vers, car il se sait bien mauvais poète. Est-ce que le cygne saurait se transformer en oie? Le hibou se mesure-t-il contre l'oiseau de Jupiter? ${ }^{54}$ Ce badinage laisse sans doute transparaître une résignation enjouée, fortement ironique, qui reflète l'attitude d'un auteur engagé dans la vie administrative et pour lequel la poésie constitue, au mieux, un divertissement à ses heures de loisir. La référence à Homère, "source et père" de tous les poètes trahit une nouvelle fois la conscience littéraire du poète qui emploie une métaphore inspirée de Quintilien pour décrire les limites même du grand poète ancien. ${ }^{55}$ Il s'agit là d'une réplique

53 Jacobi Alneti vindocinensis Epigrammata (voir la n. 1), f. 22v: "Si le for intérieur n'est pas libre de soucis, / Et si l'esprit, ayant chassé les nuages, n'est pas serein, / À peine le grand Homère lui-même / (Père et source, dit-on, de l'inspiration) / Pourra-t-il produire ses vers, quand bien même il vivrait toutes les années de Nestor."

54 Jacobi Alneti vindocinensis Epigrammata (voir la n. 1), f. 22v: "At tu si vir amice rursus instes, / Persuadere volens bonum Poetam, / Qui cantor malus est, inopsque cantus, / Qui plectri rudis, inscius lyraeque est, / Transformentur in Anseres olores, / Et sit Noctua par Iovis volucri." ("Mais si toi, mon ami, tu t'efforces une nouvelle fois / De convaincre, par bienveillance, qu'il est bon Poète / Celui qui n'est qu'un mauvais chanteur au cri chétif, / Celui même qui ne sait pas manier le plectre, ni la lyre, / Puissent donc les cygnes se transformer en oies, / Et le hibou rivaliser avec l'oiseau de Jupiter.")

55 Quint. Inst. 10.46: "Hic enim, quemadmodum ex Oceano dicit ipse omnium amnium fontiumque cursus initium capere, omnibus eloquentiae partibus exemplum et ortum dedit." 
complémentaire à la méditation sombre que proposait Delaunay sur le mode d'un adieu à la poésie.

Même dans les premières épigrammes de cette petite série, où Jacques Delaunay loue la liberté idyllique dont jouirait le poète monastique affilié à la maison abbatiale de Molesme, la réalité qui sous-tend l'échange semble bien être celle de l'absence de véritables loisirs poétiques. Ensuite, dans le dernier poème de l'échange entre les deux hommes, une épigramme, de trente-quatre vers constitués d'hexamètres suivis de sénaires ïambiques en alternance, adressée par Delaunay à Marconville, l'auteur finit par reconnaître que son destinataire mène, lui aussi, une existence qui laisse bien peu de place au culte des muses. Il ouvre le poème en lui demandant s'ils ne vont pas, tous les deux, "abandonner la forêt de Pégase" sous les diverses pressions que la vie active exerce sur eux. Que valent les muses dans un tel contexte? Ne vont-ils pas devoir arrêter de chanter? Delaunay apporte lui-même une première réponse, pessimiste, à cette question en expliquant que les réalités de la vie laissent peu de choix aux poètes sans mécène:

Heu sic fata volunt, quoniam distringimur ambo;

Tu litium fasce, ego medendi angoribus.

Ut pereant morbi et morborum causa Prometheus,

Qui primus illos intulit mortalibus;

Utque adeo superi perdant litesque forumque,

Et quae parens est litium Philautia. ${ }^{56}$

Or, l'auteur affirme à la conclusion de son poème que des deux professions qu'ils exercent parallèlement, celle de son correspondant monastique est la plus dure, la plus démoralisante. Alors que le médecin Delaunay recueille quelque fruit de ses labeurs incessants, le juriste Marconville s'investit pleinement, de façon ingrate, dans les dossiers qu'il gère. Delaunay laisse entendre qu'il y aurait même quelque danger inhérent à ce type de combat qui exposerait l'intéressé aux affres de la lutte juridique et administrative. Son emploi du substantif Philautia, qui désigne manifestement un personnage allégorique, le rapproche d'un

56 Jacobi Alneti vindocinensis Epigrammata (voir la n. 1), f. 22v: "Hélas! tel est le vouloir des destins, puisque nous sommes retenus tous les deux, / Toi, par le faisceau des litiges; moi, par les préoccupations de la médecine. / Puissent-ils périr, les maladies et Prométhée cause des maladies, / Lui qui le premier les infligea aux mortels, / Et puissent les dieux eux-mêmes perdre leurs litiges et leurs procès, / Y compris Philautie, la mère des litiges." 
passage semblable dans le Zodiacus vitae, où Palingène réfléchit aux pouvoirs limités des être humains dans leur quête de comprendre les mystères de la Nature. ${ }^{57}$ Afin de mettre Marconville en garde contre des ennuis éventuels, il déploie à la fin du poème un exemple puisé dans la fable ésopique sur le lion, l'ours et le renard. ${ }^{58}$ Dans cette fable célèbre, l'observateur d'un litige au forum intervient à la fin du conflit, lorsque les deux adversaires, tels le lion et l'ours épuisés par l'effort et affaiblis par leurs blessures, se trouvent désormais incapables de combattre. Delaunay dresse ainsi, pour caractériser la vie active que mène Marconville, un tableau allégorique plutôt sombre qui se déteint sur l'ensemble de leur échange.

L'échange poétique entre Delaunay et Marconville révèle, malgré le ton parfois sombre qui transparaît surtout chez le premier, une préoccupation commune avec les exigences de la vie active et la difficulté de composer des vers pour ceux qui ne disposent pas de la marge de liberté nécessaire aux œuvres de l'esprit. Il s'établit à travers ces pièces un rapport thématique subtil entre l'idée d'une vie consacrée à la contemplation spirituelle et celle d'une existence dévouée à la composition des vers. Lorsque Delaunay déclare son intention d'abandonner la poésie en affirmant qu'elle ne lui a jamais apporté gains ni bénéfices matériels, il évoque à sa manière une relation d'exclusivité, voire d'incompatibilité, qui subsiste entre la vie active et la vie des lettres qu'il associe manifestement à la vie contemplative. Cette opposition fondamentale évoquée par les deux correspondants, dont les origines littéraires remontent bien au-delà des méditations de Pétrarque sur le même thème dans le De vita solitaria et le Secretum, se manifeste ici dans un contexte où les poètes expriment tous les deux leur difficulté à concilier les exigences de la vie pratique et de l'otium poétique. ${ }^{59}$

\section{Jean Dampierre}

Le recueil de Jacques Delaunay contient aussi un échange de poèmes entre l'auteur et Jean Dampierre, poète monastique proche des milieux

\footnotetext{
57 Palingène Zodiacus vitae 6.191-193: "Stultitiae fons est et origo philautia vestrae, / Caligoque ingens, quae vos cognoscere verum / Posse vetat...".

58 Jacobi Alneti vindocinensis Epigrammata (voir la n. 1), f. 22v.

59 C. Trinkaus, The Poet as Philosopher: Petrarch and the Formation of Renaissance Consciousness (New Haven, 1979).
}

Humanistica Lovaniensia 70.2 (2021), 155-183 
littéraires d'Orléans et de Lyon. ${ }^{60}$ Cet échange, qui apparaît vers la fin du recueil à la suite de celui entre Delaunay et Marconville, constitue, comme ce dernier, une partie importante du recueil en raison de la longueur relative des pièces. La séquence contient quatre poèmes au total, deux de chaque auteur. Elle laisse transparaître une relation amicale entre les deux hommes, marquée par l'attitude de déférence admirative dont fait preuve Delaunay à l'égard de son correspondant. Celui-ci se montre toutefois fort respectueux du métier et des connaissances du médecin, intérêt personnel qui se manifeste avec éclat dans ses autres œuvres.

Une longue épître de Dampierre composée en hendécasyllabes ouvre l'échange, dans laquelle il se présente bien modestement à Delaunay en admirateur de ses vers. Il lui explique qu'un ami commun vient de lui montrer un échantillon de ses écrits qu'il a trouvés à la fois édifiants et "pleins de charme". Plus loin, vers la fin du poème, Dampierre reconnaîtra discrètement que c'était bien Delaunay qui lui transmettait ses vers par l'intermédiaire d'un certain "Paul", de ses amis, dont le nom de famille n'est pas précisé. Cette longue pièce épistolaire constitue donc en réalité la réponse de Dampierre à un envoi initial de l'auteur du recueil. Le maître de la forme hendécasyllabique, membre éminent des milieux lettrés actifs à Orléans, réagit ainsi amicalement à l'épître du médecin et chanoine de Saint-Étienne de Troyes.

Cette ouverture de six vers jette donc une certaine lumière sur l'état des relations entre les deux hommes qui se connaissent uniquement, à ce qu'il semble, de réputation et par leurs écrits. Fidèle au protocole rhétorique des bons rapports humanistes, Dampierre fait tout d'abord, dans les premiers soixante-cinq vers, l'éloge de son destinataire. Dampierre élabore sur un ton enjoué son éloge de Delaunay à partir d'une description détaillée de l'arbre qui forme la racine de son nom de famille ("alnus"). Ce procédé lui permet de montrer non seulement sa prouesse dans le maniement des hendécasyllabes, mais aussi ses capacités inventives en matière de description naturelle, pratique dont il fait abondamment preuve aux deux premiers livres de son traité sur l'éducation des jeunes filles. Bien que l'aulne soit un arbre stérile, explique-t-il, qui ne livre ni fruit ni grain, les produits que donne cet arbre particulier - le poète Jacques Delaunay lui-même - s'écartent de la norme

${ }^{60}$ Voir sur cet auteur les articles cités de Pédeflous 2009 (voir la n. 9) et Meylan 1976 (voir la n. 10) ainsi que l'article ancien de J. Boussard, "Un poète latin directeur spirituel au XVIe siècle: Jean Dampierre", Bulletin philologique et historique, 1946-1947, 33-58. 
en raison de leur valeur exceptionnelle. Ici, Dampierre n'hésite pas à déployer l'ironie typique de l'éloge paradoxal:

Tua inquam mihi scripta Paulus ille

Ostendit, dubio procul venusta,

Et quanquam artifice ex eo profecta,

Cui nomen tribuit locus palustri

Alno consitus arbore, arbore, inquam,

Nec fructum, neque semen adferente,

Sed nec aedibus utili struendis,

Nimirum fragili, parumque firma. ${ }^{61}$

Ce début d'éloge peu conventionnel est suivi d'un long développement sur les qualités particulières de l'aulne qui, sans ressembler aux arbres plus attrayants et fructifères, s'avère néanmoins richement fécond en poésie. Soulignant le fait que les aulnes croissent généralement dans des bois humides, en des lieux marécageux ou sur le bord des cours d'eau, ${ }^{62}$ Dampierre identifie dans ce trait naturel de l'arbre un caractère analogue à la poésie de Delaunay. Enseveli sous le sol ou submergé près d'une rive, ${ }^{63}$ conditions qui mèneraient $d$ 'autres arbres au décès et à la putréfaction, l'aulne demeure étonnamment vigoureux pendant des siècles. C'est en filant ainsi la métaphore de l'existence peu glorieuse, mais noblement insolite, de l'aulne, que l'auteur construit sa réflexion sur l'œuvre de celui qui en porte le nom. Si en effet l'aulne ne donne ni fleurs ni fruits, il demeure néanmoins un arbre important en raison de sa grande utilité à l'homme:

\section{Ob idque}

Quaecunque in mediis struuntur undis,

Fundamenta iaci alnea his necesse est.

Omninoque liquet, latentem in undis

Vel tectam sub humo integram atque sanam

61 Jacobi Alneti vindocinensis Epigrammata (voir la n. 1), f. 26r: "Tes écrits, dis-je, l'ami Paul me les a / Présentés, décidément pleins de charme. / Mais cependant produits par un artisan / Qui reçoit son nom du lieu planté / De l'aune, arbre des marécages, arbre, dis-je, / Qui n'apporte ni fruit, ni semence, / Et qui n'est pas utile pour construire des édifices, / Fragile, en effet, et trop peu solide."

62 Cf. Verg. Georg. 2.451; Lucan. Phars. 3.441; Stat. Theb. 6.106; Claud. Hon. IV 4.422 .

63 Cf. Verg. Georg. 2.110: "Fluminibus salices crassisque paludibus alni / Nascuntur...". 
Alnum vivere saecla multa. Quodque

Consuevit putrefactionis esse

Multis causa, perennitatis huic est... ${ }^{64}$

Cette surprenante pérennité de l'arbre annonce aussi, selon Dampierre, le sort bienheureux, insoupçonné de ses contemporains, d'un auteur d'humbles épigrammes latines. Sans offrir de louanges dithyrambiques, l'éloge paradoxal que profère ici Dampierre exprime le soutien, et une certaine solidarité, du poète spécialiste d'hendécasyllabes. Les vers de Jacques Delaunay seront sans doute immortels, suggère-t-il, autant pour leur élégance savante qu'en raison de la robuste utilité de leur propos. Ce caractère savant transparait notamment de l'emploi d'un terme rarement attesté en poésie comme putrefactio, qui reflète plutôt une connaissance d'œuvres scientifiques comme celle de Pline l'Ancien. ${ }^{65}$ A travers cette figure de l'arbre, Dampierre se fait l'apologiste à la fois ludique et raffiné des genres poétiques mineurs dont il est lui-même un praticien fort expérimenté et largement reconnu. Il adhère ainsi, à son tour, à la réflexion commune sur la dignité des petits genres qui traverse en filigrane tout cet échange.

De l'éloge du poète, Dampierre passe ensuite à celui du médecin qu'est également Delaunay, domaine qui suscite tout spécialement l'intérêt du moine érudit. Dans les deux premiers livres du long traité en hendécasyllabes que signe celui-ci sur le thème de l'éducation des jeunes filles, le lecteur rencontre en effet, non quelque sermon édifiant sur la valeur morale de la bonne conduite féminine, mais bien un véritable traité de médecine et de physionomie, truffé de références à Galien et à Hippocrate. Il n'est donc pas surprenant de rencontrer, dans cette épître en vers adressée au médecin Delaunay, une louange passionnée de l'art médical ancien et moderne, suivi en dernier lieu d'un nouvel éloge de ses poésies qui, déclare-t-il, constituent un fruit précieux et durable ${ }^{66}$. Il conclut son épître sur une péroraison énergique de trente-trois vers, proposant à son destinataire de s'appeler de bons amis directement et sans

64 Jacobi Alneti vindocinensis Epigrammata (voir la n. 1), f. 26v : "Et c'est pourquoi / Toutes les constructions situées au milieu de l'eau / Doivent s'appuyer sur des fondations en bois d'aulne. / Et il est tout à fait limpide que, même cachée sous l'onde / Ou recouvert de terre, l'aulne vit sain / Et en parfait état de nombreux siècles: ce qui / D'habitude, chez beaucoup, est cause de la putréfaction, / Le rend, au contraire, pérenne..."

65 Plin. M. NH 29.90.

66 Jacobi Alneti vindocinensis Epigrammata (voir la n. 1), f. 26v-27r. 
formalité, tant il ressent de la sympathie pour le savant poète et médecin originaire de Vendôme. ${ }^{67}$

Cette longue pièce en hendécasyllabes, à travers laquelle Jean Dampierre tend une main amicale à Jacques Delaunay, est suivie de la réponse de celui-ci, une pièce de soixante-huit vers composée en strophes alcaïques. L'auteur assume d'emblée une posture d'humilité, cherchant à illustrer le décalage entre la médiocrité de ses vers et la virtuosité éloquente de l'éloge qu'il vient de recevoir:

Ut indecore rusticus applicet

In lente nardum, bubus ephippia,

Suesque donentur smaragdis

Turpiter, et phaleris capellae;

Sic indecenter me celebras rudem,

Cantu beato divitis ingeni,

Me quem nec artibus decorat

Melpomene, nec Apollo lauris. ${ }^{68}$

L'incongruité des associations désignées dans ces vers, entre l'humble lentille et le riche nard, entre la couverture de cheval de race et le rude bétail figure celle des compliments qu'adresse Dampierre à son correspondant et admirateur. Cette fois encore, l'usage de termes comme ephippia, mot grec attesté plutôt chez les prosateurs, ${ }^{69}$ renforce un certain réalisme ludique propre à alimenter le caractère souriant et amical de l'éloge enjoué qui fait preuve également de la virtuosité qu'exige un lexique ainsi insolite et enrichi. Delaunay reconnaît volontiers, à travers une série d'images qui lui permet, à la fois, de montrer sa compréhension du procédé encomiastique adopté par Dampierre et d'exhiber discrètement ses propres aptitudes à la composition de strophes lyriques, le sous-texte de l'éloge parfois ironique élaboré dans le poème du moine. Alors que celui-ci déclare que l'aulne emblématique de son nouvel ami

67 Jacobi Alneti vindocinensis Epigrammata (voir la n. 1), f. 27r-v.

68 Jacobi Alneti vindocinensis Epigrammata (voir la n. 1), f. 27v-28r: "De même que le paysan attacherait incongrûment / Le nard à la lentille, la selle aux vaches, / De même que les porcs seraient gratifiés indécemment / D'émeraudes, et les chèvres de phalères, / C'est d'une manière tout aussi déplacée que tu me fêtes, rustre que je suis, / Par un chant bienheureux d'inspiration riche, / Moi que ni Melpomène n'honore / De ses artifices, ni Apollon de ses lauriers."

${ }^{69}$ Cf. Cic. Fin. 3.15: "Et tamen puto concedi nobis oportere ut Graeco verbo utamur, si quando minus occurret Latinum, ne hoc 'ephippis' et 'acratophoris' potius quam 'proëgmenis' et 'apoproëgmenis' concedatur." 
s'abreuve abondamment des ondes de Castalie, Delaunay lui-même use de ces mêmes images pour se caractériser comme un poète médiocre, dépourvu de l'inspiration des muses.

Cette posture d'humilité, où le poète affecte de reconnaître la médiocrité de son talent, constitue ainsi un motif récurrent des échanges que poursuit Jacques Delaunay avec ses confrères poètes, préservés au moins en partie dans le recueil de 1539. Entre Jean Dampierre et luimême, il note une séparation d'ordre hiérarchique comme en témoignent ses fréquentes protestations de son indignité et de sa petitesse face au destinataire. Trop lourde lui paraît la charge de chanter l'éloge de ce grand homme de lettres qui appartient de bon droit à la société raréfiée des poètes-nés, dotés de l'inspiration surnaturelle. Delaunay revient sur le thème de l'arbre introduit par Dampierre, décrivant la terreur qu'il ressent devant le poète vénéré:

Quid tu? Quid, heros Pieriae domus?

Qua laude, quo te carmine dixero?

Haeresco, mique magnitudo

Obserat os adimitque vocem.

Nuper sedebam stipes inutilis

Exclusus almo fonte lybetridum.

Furtim tamen Phoebum et Camenas

Auribus atque oculis notabam.

Eho boni Dii, quas ego tibias

Tibi sonanteis magnificum audii?

Nephas, nephas, mihi deorum

Verba rudi recitare plectro. ${ }^{70}$

Un jeu de sonorités ("Pieriae domus") évoque déjà le nom du moine destinataire. L'expression autodépréciative "stipes inutilis" nourrit ensuite la réponse modeste aux propos amicaux de Dampierre, notamment à la métaphore filée de l'aulne durable et exceptionnellement fécond,

70 Jacobi Alneti vindocinensis Epigrammata (voir la n. 1), f. 28r-28v: "Comment? toi? Le héros de la maison des Piérides? / Avec quelle louange, avec quel chant te décrirai-je? / Je reste figé, la grandeur de la tâche / Me clôt la bouche et me prive de voix. / Naguère je demeurais, arbre inutile, / Exclu de la source bienfaisante des Libéthrides. / En cachette toutefois je captais Phébus et les / Camènes des oreilles et des yeux. / Holà! dieux bons, quelles flûtes ai-je / Entendues, qui faisaient entendre de nobles mélodies pour toi? / Un sacrilège, c'est bien un sacrilège pour moi / De réciter les mots des dieux aidé d'un plectre grossier." 
développée longuement dans la pièce précédente. Un écho virgilien transparaît de l'expression ici usitée ("Haeresco...") pour décrire le sentiment de frayeur qui réduit le poète au silence devant celui qu'il admire tant. ${ }^{71}$ Delaunay déclare ensuite avoir témoigné d'une scène imaginaire, où le chœur des Thespiades est venu fêter joyeusement la gloire éternelle du maître Dampierre, poète nouvellement couronné d'Apollon. ${ }^{72}$ Il termine l'ode en soulignant à nouveau l'idée d'une relation hiérarchique entre lui-même et son correspondant, exprimant avec ferveur sa gratitude envers ce grand poète qui daigne le recevoir parmi ses amis proches au sein de la république des lettres. ${ }^{73}$

La pièce suivante est signée Dampierre et comporte sa réaction à la réponse de Delaunay. Composée toujours en hendécasyllabes, cette épigramme de 77 vers livre un nouveau message d'admiration pour le médecin et poète qui habite Troyes, renouant brièvement avec la métaphore de l'arbre et de la fertilité développée dans les deux poèmes précédents. Dampierre déclare tout d'abord qu'il ne peut offrir à Delaunay rien d'autre qu'une affection sincère, le seul don de l'amitié véritable. Il craint toutefois que l'amitié indéfectible qu'il portera à Delaunay ne soit "stérile" pour celui-ci ("Sed tibi sterilis veremur, ut sit"). Sachant déjà, en revanche, que l'affection et le commerce personnel du médecin Delaunay lui sera bien profitable, son intention, déclare-t-il, est de profiter humblement de la sagesse et des connaissances de son ami:

At decerpere plurima ex amore

Alneti bona praeter ipsum amorem, Possit Dampetrus. Utpote edoceri

Qui rudis queat a viro erudito.

Nempe praecipuum ex suis amicis

Hunc captare suevit, ille fructum;

Ut cum labitur (id frequenter autem

71 Cf. Verg. Aen. 2.77 ; et 3.48 "Obstipui steteruntque comae et vox faucibus haesit." ainsi que Aen 4.280 et 12.868 ("arrectaeque horror comae et vox faucibus haesit").

72 Jacobi Alneti vindocinensis Epigrammata (voir la n. 1), f. 28v.

73 Jacobi Alneti vindocinensis Epigrammata (voir la n. 1), f. 29r: "O vive felix nobilium decus / Scientiarum et dimidium mei. / Te, sive Creusulus beatus vixero, sive miser colonus, / Nostris habebo sensibus intimum, / Totus virebo, si vireo, tibi. / Pro te periculum tremendum / Non metuam, nec obire mortem." ("Ô gloire vivante et bienheureuse des nobles / Savoirs et la moitié de moi-même! / Que je mène mon existence en petit Crésus / Bienheureux, ou en pauvre paysan, / Je te garderai au fond de moi-même. / Si je m'épanouis, c'est pour toi que je m'épanouirai tout entier. / Pour toi je ne craindrai de braver ni le péril / Redoutable, ni même la mort.") 
Usu venit ei) repente ab illis

Libere, ingenueque corrigatur.

Dein si coeperit aeger esse forsan,

Quod non ille perinde consuevit:

Praescriptis adeo salubris artis

Alnetus medicus iuvare possit. ${ }^{74}$

Tel le rustre qui se laisse éduquer par un savant, Dampierre entend se faire corriger par le poète médecin. Cette déclaration d'humilité rapproche l'auteur, quant à son expression poétique, du prologue de la deuxième Fable de Phèdre, qui explique aux lecteurs la profonde valeur pédagogique des Fables d'Ésope, qui exigent une lecture avertie. ${ }^{75} \mathrm{La}$ nouvelle mention de la profession de Jacques Delaunay reflète encore une fois l'intérêt réel du moine pour l'étude du corps et de la santé. Son emploi de la métaphore végétale perpétue discrètement le réseau d'images développé dans les deux premiers poèmes de l'échange. Dampierre s'interroge ouvertement sur ce que pourront être les bénéfices de leur amitié dans la perspective de Jacques Delaunay:

Quid ergo tibi commodare possim,

In quo non magis usui esse nobis

Possis? Ni tibi forsitan Sacerdos

Desit, cui tua more Christiano

Ut fit, crimina detegas in aurem.

(Haec nostri officii una nempe pars est,

Si nescis, et ea haud levis negoci.) ${ }^{76}$

74 Jacobi Alneti vindocinensis Epigrammata (voir la n. 1), f. 29v: "Or cueillir plusieurs avantages de l'amour / De Delaunay, sans entamer l'amour même, / Dampierre le pourrait. Puisque le rustre / Peut se faire éduquer par un homme savant, / Tel est le fruit principal que celui-ci a pris l'habitude / De glaner chez ses amis: / Que, lorsqu'il glisse dans l'erreur (chose qui lui arrive / Fréquemment en effet), se faire spontanément, / Librement, honnêtement corriger par eux. / Ensuite, si par hasard il tombe malade, / Ce qui par ailleurs ne lui arrive pas souvent, / Avec les ordonnances de son art de guérir / Le médecin Delaunay pourrait bien l'aider."

75 Phaedr. Fab. 2.prol.1-4 : "Exemplis continetur Aesopi genus; / Nec aliud quicquam per fabellas quaeritur / Quam corrigatur error ut mortalium, / Acuatque sese diligens industria."

${ }^{76}$ Jacobi Alneti vindocinensis Epigrammata (voir la n. 1), f. 30r: "Que pourrais-je donc t'offrir, / En quoi tu serais moins efficace que / Je ne le suis? A moins que par hasard il ne te faille / Un prêtre, à l'oreille duquel tu puisses, / En bon Chrétien, révéler tes péchés. / (Ceci fait partie de mon office, n'est-ce pas? / Si tu ne le sais, et ce n'est guère une mince affaire.)" 
En échange de la sagesse et du savoir dont le poète médecin pourra lui faire bénéficier, le poète monastique promet d'offrir les fruits de son dévouement et de son office religieux au cas où l'ami aurait besoin de la confession. Une bonne part du ton d'humilité qui transparaît de ces vers réside dans la suggestion d'un rapport d'équivalence qui subsisterait entre la médecine du corps et les soins de l'âme. Le moine conclut même, par modestie, que ses dons sont finalement peu de chose en comparaison avec ceux de son ami, mais il se rassure du fait que l'amitié véritable demeure étrangère à des considérations d'échange ou de profit.

Dans la pièce qui clôt cet échange versifié entre Delaunay et Dampierre, l'auteur du recueil revisite une dernière fois les topiques de l'amitié désintéressée et de la modestie, déjà élaborées au cours des compositions qui précèdent. Décrivant avec précision le contraste qu'il voit entre Dampierre et lui-même, Delaunay reproche à celui-ci de ne pas reconnaître dans sa propre personne les qualités qui font un homme d'exception. Il introduit une dernière fois le motif de l'aulne humble et rampant:

Me ne tibi aequiparas? Picam iam confer olori.

Et misce tenebris hac ratione diem,

Alnus humi serpit metuens se attollere terra:

Praepetibus pennis tu per inane volas.

Haereo naturae, corruptaque corpora tantum

Verso animo, necdum me reor esse satis.

Tu res aeternas, atque omni faece carentes,

Quaeque oculis non sunt subdita, mente capis.

Lingua rudis nobis, et iners infantia torpet,

At tibi dicendi vena beata fluit.

Adde quod iratis musis et Apolline canto,

Tu Phoebi plenum numine pectus habes.

Denique nostra opera est hominum succurrere morbis

Tu curas animos: scisque docesque Deum. ${ }^{77}$

77 Jacobi Alneti vindocinensis Epigrammata (voir la n. 1), f. 31r-v: "Me mets-tu vraiment à ton niveau? Autant comparer la pie et le cygne / Et, suivant cette logique, confondre la nuit et le jour. / L'aulne rampe sur le sol, craignant de se lever de la terre; / Quant à toi, d'une aile rapide tu voles à travers l'espace. / Je m'accroche à la Nature, les corps corrompus seulement / J'observe, ne me considérant pas encore suffisant. / Toi, tu saisis de ton esprit les choses éternelles, qui manquent de toute / Souillure, et que les yeux ne sauraient voir. / Ma langue est rude, et inerte, elle languit sans parler, / Alors que chez toi coule une veine bienheureuse de paroles. / Ajoutons que je chante au grand dam et à la 
D'après la description que donne ici Jacques Delaunay, tout semble séparer les deux hommes. La comparaison de la pie et du cygne évoque la réflexion satirique et métalittéraire de Persius qui a suscité des imitations chez les épigrammatistes de la fin du Quattrocento. ${ }^{78}$ Alors que le grand Dampierre, doté de la fureur apollinienne, hante les régions célestes, son ami rampe sur le sol, craignant de se lever. Si le médecin s'accroche à l'étude de la nature et des corps destinés au néant, le moine contemple les choses éternelles et sans corruption. Alors que Delaunay compose ses vers contre la volonté à la fois des muses et de leur maître Apollon, Dampierre chanterait les siens sous le mouvement d'une inspiration divine.

La correspondance poétique des deux humanistes tourne, on le voit, au jeu de l'esprit et au concours d'éloges, chacun déployant les lieux communs de l'humilité pour décrire avec éloquence son rapport à l'autre. De tels éloges ont beau refléter la sincérité de leur auteur, ils finissent néanmoins par s'inscrire dans le fonds traditionnel des procédés encomiastiques, dont les traits particuliers constituent, on le sait, une véritable matière d'étude pour les humanistes. ${ }^{79}$ Mais à la fin de leur échange, une légère altération de ce discours badin, introduite en dernier lieu par Jacques Delaunay, vient ponctuer l'ensemble. Après avoir évoqué ici encore la métaphore de l'aulne emblématique de son œuvre, Delaunay met abruptement fin à ce jeu dont il reconnaît explicitement enfin le caractère ludique, en soulignant la vanité de la joute des éloquences rivales: "Mais à quoi bon? Pourquoi lançons-nous tant de mots / De l'un à l'autre, pour savoir qui de nous deux doit passer devant l'autre?"80 Aussi exhorte-t-il Dampierre à reconnaître avec lui que l'amitié se construit sur un fonds commun, malgré les différences superficielles d'aspect et de talent qui peuvent séparer les hommes. La distinction de leurs modes de vie et de leurs vocations ne suffit pas en effet à les scinder et les rendre proprement étrangers l'un à l'autre. Ils se doivent alors, au nom de l'affection amicale, d'assumer leur rapport d'égalité afin de nourrir le sentiment de réciprocité qui les unit. Dans ce discours sur l'amitié sincère

colère des Muses et d'Apollon, / Alors que toi, tu as le cœur rempli de la puissance de Phébus. / Enfin mon office est de secourir les maladies des hommes, / Toi, tu soignes les âmes; tu connais Dieu et tu l'enseignes."

78 Pers. Prol. 8-14; Panfilo Sasso Epig. 1, 61, 79.

79 P. Galand, "Jean Salmon Macrin et la liberté de l'éloge", in Luisa Rotondi Secchi Tarugi (ed.), Cultura e potere nel Rinascimento (Florence, 1999), 515-529.

80 Jacobi Alneti vindocinensis Epigrammata (voir la n. 1), f. 32r: "Sed quid ego haec? Cur nos ultro tot verba citroque / Caedimus, alter utro debeat esse prior?" 
et profonde, Delaunay introduit soudain sous un nouveau jour la double métaphore de l'arbre et du fruit:

Quin agedum unanimes vero incumbamus amori,

Quem vel latum unguem non movet utilitas.

Verus amicitiae fructus consensus honesti est

Nec nisi in interno corde triumphat amor.

Qui postquam inter nos XPO duce et auspice coepit,

Ut puto, radices altius intus aget.

Quem si morte putas finiri posse, negabo.

Utque negem ratio, firmaque causa movet.

Immortale bonum est animi immortalis amare,

Ergo bonus noster nec moriturus amor. ${ }^{81}$

Le réseau d'images propre à la métaphore végétale, qui comprend l'arbre, ses racines et le fruit qu'il donne, s'accompagne ici de références à l'intériorité ("interno corde", "altius intus aget") et à la sincérité ("vero...amori", "verus amicitiae fructus") du rapport amical qui liera ces deux hommes passionnés de la science médicale, de la poésie et de la foi chrétienne. Chez les poètes latins de l'Antiquité, l'épithète "internus" apparaît tardivement, notamment chez les poètes chrétiens. Cette référence à l'intériorité chez Delaunay n'est pas sans ressembler à la réflexion analogue attesté dans l'épopée de Juvencus, qui paraphrase, en l'amplifiant de façon à développer largement le motif, la remarque de Jésus dans Matthieu 15:11, selon laquelle "ce n'est pas ce qui entre dans la bouche qui profane l'homme: mais ce qui sort de la bouche, voilà ce qui profane l'homme." 82 Une juxtaposition aussi forte de ces thématiques connexes, l'intériorité et la sincérité, associées de près au registre métaphorique développé tout le long de ces quatre pièces, amène aussi l'accomplissement de leur échange à la fois affectueux et ludique. La

81 Jacobi Alneti vindocinensis Epigrammata (voir la n. 1), f. 32r: "Eh bien, allons d'un même esprit nous engager dans un véritable amour / Qui pas le moins du monde ne s'inspire de l'intérêt. / Le véritable fruit de l'amitié est l'accord dans la vertu, / Et c'est seulement dans le fond du cœur que l'amour triomphe. / Une fois qu'entre nous sous la conduite et les auspices du Christ il a commencé, / A mon avis, il poussera plus profondément ses racines à l'intérieur, / Et si tu penses que la mort pourra y mettre un terme, je le nierai, / Négation motivée par une raison et une cause solide: / L'amour est un bien immortel qui est le propre de l'âme immortelle, / Voilà pourquoi notre bon amour ne mourra jamais."

82 J. Grosjean et al. (ed.), La Bible. Nouveau Testament (Paris, 1971), 51. Cf. Juvencus Evang. 3.166-170. 
figure descriptive de l'aulne, introduite longuement par Jean Dampierre dans la première pièce de leur correspondance, en acquiert une valeur symbolique renouvelée, renforcée notamment par la mise en valeur de l'image des racines nourricières qui alimentent le "fruit" de la véritable amitié. Jacques Delaunay réaffirme ainsi la valeur de l'éloge paradoxal, voire la défense et illustration symbolique des objets humbles, que Dampierre s'employait à construire déjà dans ses premières références à ce motif antique. Aussi finit-il par faire déplacer leur échange de poèmes épistolaires vers un terrain plus intime que n'est celui des premiers badinages inventifs qui ouvrent leur brève correspondance poétique préservée dans les Epigrammata de 1539.

\section{Conclusion}

Ces échanges poétiques entre Jacques Delaunay et ses amis Nicolas de Marconville et Jean Dampierre livrent un témoignage supplémentaire de la vitalité des milieux lettrés pendant le règne de François Ier. Chez les trois auteurs, la proximité affichée aux instances ecclésiales et monastiques constitue également un trait particulier qui les distingue des contemporains à l'orientation plus profane, surnommés les "Apollons de Collège". Car ceux-ci sont en effet plus fortement influencés par les modèles épigrammatiques de Martial et Catulle. ${ }^{83}$ Dans le contexte même de cette affinité déclarée aux institutions sacrées, la correspondance qu'entretient Delaunay avec Marconville reflète une préoccupation continuelle avec les exigences de la vie active et les pressions croissantes que celle-ci peut exercer sur le loisir que réclament les belles-lettres. Au cours de leur échange, ils donnent voix à l'idée que certains genres poétiques mineurs, comme l'épigramme, conviennent au poète dont les œuvres modestes sont le fruit des heures de loisir. Un ton d'humilité parfois teintée d'ironie caractérise semblablement l'attitude adoptée par le médecin Delaunay dans sa correspondance poétique avec Dampierre. Ici, le motif cicéronien de l'amitié cher aux humanistes constitue un terrain de rencontre où les deux auteurs déploient leurs réserves d'éloquence. Cet échange est marqué par la virtuosité descriptive dont fait preuve Dampierre dans ses mentions détaillées de l'aulne, arbre à la fois humble

83 C. Langlois-Pézeret, "Gilbert Ducher, Apollon d'Aigueperse, poète et principal de collège dans les années 1530 à Lyon", in M. Ferrand, N. Istasse (ed.), Nouveaux regards sur les 'Apollons de Collège'. Figures du professeur humaniste dans la lère moitié du XVIe siècle (Genève, 2014), 221-222. 
et honorable qu'il associe de façon enjouée à l'identité de son correspondant. Leur dialogue reflète également une attitude de respect réciproque animant les rapports entre les deux hommes, le médecin et le moine, qui évoquent à plusieurs reprises leurs vocations dans la vie "active". Cette affection naissante, déclare Delaunay, puisera sa vitalité dans la foi qu'ils partagent et qui en garantit la vertu désintéressée. Enfin, dans les relations qui se construisent à travers ces échanges entre Jacques Delaunay et ses confrères Marconville et Dampierre, l'amitié s'abreuve généreusement à la source de l'amour des lettres tant sacrées que profanes. Une telle conviction partagée demeure ancrée chez les trois hommes dans l'éthique chrétienne qui mélange volontiers l'inspiration divine et l'amour du prochain, la découverte personnelle et le don amical.

Western University jnassich@uwo.ca 